\title{
Exploration of Grade 9 Learners' Errors on Operations with Directed Numbers
}

\author{
Judah Makonye (Dr) \\ School of Education, University of Witwatersrand, Number 27 St Andrews Road \\ Parktown, 2193, Johannesburg, South Africa \\ Email: Judah.makonye@wits.ac.za
}

Nwabisa Hantibi (Mrs)

Kensington Secondary School, corner Protea and New York roads, Kensington, Johannesburg, South Africa

Email: nwabisahantibi8@gmail.com

Doi:10.5901/mjss.2014.v5n20p1564

\begin{abstract}
The study is about the investigation of the types of errors and misconceptions that the grade 9 learners have when answering questions on operations in integers. The study also explores the misconceptions responsible for the errors grade 9 learners show in their scripts as they answer questions on operations in integers. The main conceptual framework of the study is Skemp's instrumental and relational understanding. Constructivist theory of learning was also looked at. Nineteen grade 9 learners were in the sample of the study. Data was generated through written tasks on operations in integers as well interviews based on the errors that the learners made. The interviews aimed to find out how learners justified their errors. The justifications they gave helped the researchers to have insights into the misconceptions that the learners had. The types of errors found were systematic, careless, transformation, process skills and encoding errors. The misconceptions that the learners showed in the study were mainly the misconceptions of overgeneralisation, interference and meaning.
\end{abstract}

Keywords: operations, directed numbers, errors, misconceptions

\section{Background to the Study}

This study is about the errors and misconceptions Grade 9 learners have when they do tasks on operations on integers. It was done by a pair of researchers; one based at a School of Education at University of Witwatersrand, South Africa and the other, a teacher at an urban school in Johannesburg.

Learners in South African schools and elsewhere make mistakes and errors when solving mathematics problems. This may be because of misunderstanding of questions by learners or misrepresentation and misinterpretation of a mathematics question, or it can be because of carelessness by learners (Ryan, 2007). The errors that the learners make can be constant, unsystematic and systematic. Constant errors are recurring incorrect responses to a specific number combination. Cox (1975) defines systematic errors as when there is a repeatedly occurring incorrect response that is evident in a specific algorithmic computation. Learners make these errors constantly and repeatedly which shows that there is a misconception in their thinking about a particular concept. Hence, systematic errors are symptomatic of a faulty line of thinking (Luneta \& Makonye 2010).

Grade 9 learners find it difficult to understand operations in integers particularly in the context of directed numbers. In this article, we want to report on our research on the errors and misconceptions that the learners have on operations with directed numbers.

\subsection{Purpose statement}

The purpose of this study is to explore the errors and misconceptions that the learners have when answering questions on operations on integers. Therefore the following research questions were posed:

1. What are the types of errors that the learners have when answering questions on operations in integers?

2. What misconceptions do they have regarding their answers? 


\section{Literature Review and Conceptual Framework}

In the classroom, mathematical errors are important because they are the contribution of learners to the learning process (Nesher, 1987). Teachers should welcome them instead as seeing them as undesirable. There are different types of errors that the learners make when dealing with mathematics. These include systematic errors, constant errors, reading errors, comprehension errors, transformation errors, process skills errors, encoding errors and careless errors (Cox, 1975). Cox (1975) gives examples of errors which learners make with addition: $46+3=13,21+8=11,15+2=8$. The learners in these situations add the digits separately, $4+6+3=13,2+1+8=11$ and $1+5+3=8$. The learners do have relational understanding and knowledge of the algorithm of operations (addition and subtraction), but they still make systematic errors on operations with integers. Systematic errors are defined by Cox (1975) as errors that happen when there is a repeatedly occurring incorrect response that is evident in a specific algorithmic computation. Careless errors are defined as those that occur even though the learner knew (from a cognitive perspective) exactly how to get the correct answer to the question at the time the incorrect answer was given, and would be expected to give the correct answer when responding to the same question at some later time (Ryan, 2007). For example a learner can give 4 as an answer when subtracting 4 from 9 . This does not mean that the learner does not know the correct answer, it can just be a slip, when asked again the same question the learner would give the correct answer.

Transformation errors occur when the children understand what the question asks but they are unable to identify the operation, or sequence of operations, needed to solve the problem (Ryan, 2007). This happens sometimes when the learners add instead of multiplying, or when they subtract instead of dividing. Encoding errors occur when the learners see the final answer as incomplete if it is a two or three termed answer. The learners in this situation want to 'complete' the answer by making it a single value answer or a one-term answer. For example, $3 x+5$ is seen by some learners as not the final answer. They then tend to make it final by adding these 2 terms and get $8 x$.

Drews (2005) defines misconception as the misapplication of a rule, an over-or under generalisation or an alternative conception of a situation. The errors that the learners make are caused by their misconceptions about a particular concept. For example, a learner can say $2 \times 3=5$ This can be due to the misapplication of a rule (misconception according to Drews, 2005). This type of error is a systematic error in that whenever a learner is given a similar question the learner applies the rule in a wrong situation in this case adding instead of multiplying. In this situation this kind of error is caused by a misconception of overgeneralisation. They generalise that any operation is addition.

There are other types of misconceptions that we see in learners. The misconception of interference is when the previously learned skills interfere with new skills (Olivier, 1989). "Addition schema is constructed first and is well developed, thus when a question is asked about multiplication, which is more recent piece of learning, the pupil replaces it with a question dealing with earlier material" (Olivier,1989, p.11). A learner here has a misconception of interference, where addition interferes with multiplication. Misconception of meaning occurs when learners conceive open expressions as incomplete and tend to finish them (Cobb, 1994). For example, when a learner sees $3 a+5$, a learner with a misconception of meaning does not see $3 a+5$ as an answer, he sees this as incomplete and therefore has to complete it (by adding the two terms together) and get the answer as $8 \mathrm{a}$.

Skemp describes instrumental understanding as rules without reasons. He also argues that even though it is rules without reasons, it has some advantages. He says it is usually easier to understand, its rewards are more immediate and more apparent, also, because less knowledge is involved, one can often get the right answer more quickly and reliably by instrumental thinking than relational. Findon (1998) argues that instrumental understanding may lead to insights of a relational type. He claims that an attempt at relational understanding may result in instrumental understanding. Skemp argues that relational understanding is about knowing what to do and why. "The question, 'Does the learner know it?' Must be replaced with 'How well does the learner understand it?' The first question refers to instrumental understanding and the second leads to relational understanding (South African Institute of Developmental Education (SAIDE), 2007). When the mathematics is learned relationally the connected information, or the network of connected ideas is simply more likely to be retained over time than disconnected information. Relational understanding therefore has the welcome potential to motivate the learner to new insights and ideas, and the creation of new inventions and discoveries in mathematics (SADE, 2007, p 53).

\section{Research Methods}

Our project will use the descriptive resign design. The main goal of the descriptive research design is to portray an accurate profile of phenomena. In this kind of design the investigator is concerned with providing a profile of variables and how they interact (Adams and Schvaneveldt, 2001). 
The study was done with grade 9 learners at an urban school in Johannesburg, South Africa. The sample of 19 learners of both sexes aged between twelve and fourteen years of age.

\title{
3.1 Data collection
}

Data collection in this research study consisted of a written task and learner interviews. Learners were given 30 minute long tasks on operations with directed numbers. Their scripts were marked noting in particular the errors occurring in their working and the answers. The learners were then interviewed based on the errors they made when answering questions. They were asked questions like; How did you get this kind of an answer? When asking them these questions we were looking for a detailed explanation of what caused them to make a particular error. We also probed them on their explanations so that we could be obtain an insight of the misconceptions that they have regarding their answers. The questions that we asked were to take the learners through their history (misconceptions) with the phenomenon in question (errors). The term for this process is the narrative interview (Bauer, 1996). Thus when communicating in the interview the respondent must talk the most (Cohen, Manion and Morrison, 2002)). This is important for the researcher to glean data pertinent to the research. The learners were describing and explaining how they got a particular answer.

To maintain reliability and validity, the written tasks and interview were closely aligned to the research questions (Rolfe, 2006). The interviews were also audiotaped to allow accurate analysis and interpretation. The researchers strived to establish rapport with the learners to ensure that a relaxed atmosphere reigned for learners to express themselves without fear.

\section{Data Analysis}

In this analysis, participants' responses are organised in tables. Table 1 shows the questions and some responses on addition and subtraction of integers. Table 2 shows questions and some responses on multiplication and division of integers. Table 3 shows questions and some responses on the substitution of variables by integers. Table 4 shows questions and responses of learners when simplifying expressions. After each table the tasks and the responses are analysed and interpreted. This is done by showing the justifications that the learners gave for their errors. The results are then interpreted based on the theoretical framework of the study. Lastly the analysis of errors with literature based conceptual framework is done.

The tasks done had three sections: A, B and C. Section A done without using a calculator.

\subsection{Section A: Errors associated with adding, subtracting, multiplying and dividing integers}

Table 1: Tasks and participants' responses on addition and subtraction of integers

\begin{tabular}{|c|c|c|}
\hline Question Number (Section A) & Question and Answer & Some Answers Given by Learners \\
\hline A.1 & $27-29=-2$ & 2,3 \\
\hline A.2 & $-65+45=-20$ & $110,-110,-120,20$ \\
\hline A.3 & $34-16-24=-6$ & $-74,14,-9,74,-24$ \\
\hline
\end{tabular}

The correct answer to question A1 is -2. Most learners got it correct. Four learners gave 2 as the answer and one learner wrote 3 as the answer. The five learners were then asked to justify their answers.

\author{
Researcher: Learner 1 would you explain how you got answer 2 to the question? \\ Learner 1: I said $29-27=2$ \\ Researcher: But the question read $27-29$, why did you change it to $29-27$ ? \\ Learner 1: I think it's just the same as 29 - 27, otherwise there is no answer, because you can't subtract a bigger \\ number from a smaller number. \\ Researcher: Where did you learn that? \\ Learner 1: Our primary school teacher said....it can't...
}

The justification was that when you take 27 out of 29 you have 2 left. A negative sign in front of 29 was not considered. It was argued that we just swop the numbers around, and put 29 in the place of 27 , or we just say 29 minus 27 then we get 2. The one who got 3 said he made a mistake his answer is 2 . This learner thought subtraction was commutative; a misconception. Olivier (1989) argues that available research suggests that one guiding principle is 
children's erroneous conception that subtraction is commutative, that is, the order does not matter, so $6-4$ and $4-6$ are the same, or rather they have the same answer. Also when making this type of an error, students subtract the smaller number from the larger number regardless of position (Riccomini, 2005).

Response to task A2 and what students said in support of their answers

For question A2 four learners got the answer as 110.

\author{
Researcher: Learner 2, how did you get 110 for $-65+45$ ? \\ Learner 2: 65 plus 45 equals 110. \\ Researcher: What happened to the negative sign in front of 65 ? \\ Learner 2: Mam, when the negative sign is in front we don't do anything about it \\ Researcher: Why, you mean we just ignore it and pretend that it is not there when it is there? \\ Learner 2: Yes, because it is in front, it's like $65+45$, we know that the sign of 65 is positive, but it's not there we just \\ know that it's positive, so with the -65 also we see that it is there but we don't do anything about it.
}

For a -110 answer it was argued that the sign of a bigger number was taken and the numbers were added. For a 120 also the sign of a bigger number was taken, but there was a mistake in addition. For answer 20, it was argued that a smaller number was subtracted from a bigger number, because a bigger number cannot be subtracted from a smaller number.

Response to task A3 and what students said in support of their answers
Researcher: Tell me, how did you get 74 for this question?
Learner: -16 and -24 are both negative so I added them and got 40, then I added the remaining 34 and got 74 .
Researcher: So, when you add -16 and -24 you get 40 not -40 ?
Learner: mmmm..., yes madam you are right -16 and -24 gives -40 , I made a mistake my answer is -74 .

The learner who got -74 was asked to justify his answer. He said, "There are two numbers with negative signs, I added them together and got -40 . Then $-40+34=-74$, I took the sign of a bigger number and added the two numbers."

Table 2: Tasks and participants' responses on multiplication and division of integers

\begin{tabular}{|c|c|c|}
\hline Question Number (Section a Cont.) & Question and Answer & Some Answers Given by Learners \\
\hline A4 & $(-2) \times(-4)=8$ & $-6 ;-4 ; 6 ;-8$ \\
\hline A5 & $24 \times-4=-96$ & $20 ;-56 ; 92 ; 96$ \\
\hline A6 & $-2 \times-2 \times-4=-16$ & $8 ; 16 ;-8 ; 6$ \\
\hline A7 & $(-25) \div(-5)=5$ & $-5 ;-30$ \\
\hline A8 & $14 \div-2=-7$ & $7 ; 12$ \\
\hline
\end{tabular}

Response to task A4 and what students said in support of their answers

In question A4 the -6 answer was justified in this way: -2 and -4 give us -6. I then asked about the multiplication operation that is in between those numbers. One learner said she did not see it, the other learner said she forgot to use it, she only looked at the numbers and not at the operation. Some learner gave 6 as the answer, and argued that a negative multiplied by another negative we get positive, then 2 and 4 add up to 6 . Again the multiplication operation was not used. For a -8 answer, it was argued that a negative number and another negative number give us a positive number, then 2 multiplied by 4 is 8 . This one used the multiplication operation but he made an error when using the signs.

Response to task $A 5$ and what students said in support of their answers

Researcher: The answer for question A5 is -96 but you got 20, can you explain to me how you got 20.

Learner: I said 24 - 4 gives us 20.

Researcher: What did you do with the multiplication operation that is there between 24 and -4 ?

Learner: I don't know, I did not recognise it. I just saw 24 and -4 and I subtracted 4 from 24.

Researcher: (talking to another learner) I see that you got 96 instead of -96, how come?

Learner K: I multiplied 24 by 4 and got 96

Researcher: What happened to the negative sign of 4 ?

Learner K: I forgot to use the negative sign. 


\subsection{Section B: Errors associated with substitutions using integers}

Table 3: Questions and the participants' responses on the substitution of variables by integers

\begin{tabular}{|c|c|c|}
\hline Question Number (Section B) & Question and Answer If $p=-4, a=2$ and $b=-3$ find the following; & Some Answers Given by Learners \\
\hline B1 & $a b=2(-3)=-6$ & $-1,-5,1,-2,-23,(2+-3)$ \\
\hline B2 & $p+a=-4+2=-2$ & -6 \\
\hline B3 & $b p-a p=-3(-4)-2(-4)=20$ & $3,-13,-7,-9,-4,(-3-4)$ \\
\hline B4 & $a+b-p=2+(-3)-(-4)=3$ & $-5,-1,2,9$ \\
\hline
\end{tabular}

Two learners did not attempt this question saying they do not understand what they were to do. Two other learners gave answers like $4 \mathrm{ab}, 3 \mathrm{pb}$. These I would say they did not understand the question.

Response to task B1 and what the students said in support of their answers

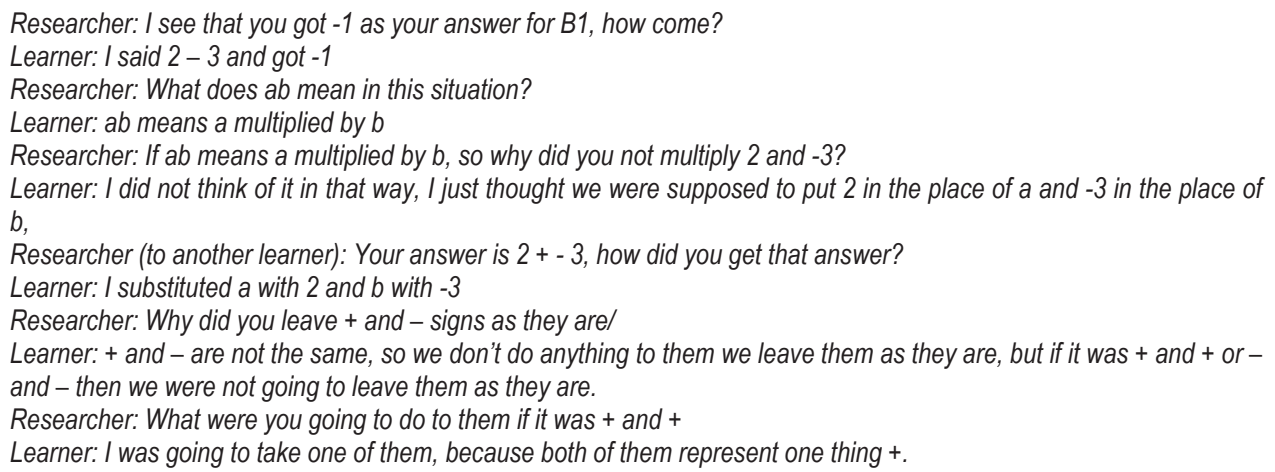

Response to task B2 and what students said in support of their answers.

The answer for B2 is -2 . Two learners got -6 as their answers. It was argued that $-4+2$ gives -6 because we take the sign of a bigger number which is negative in this case, then we add 4 and 2 together and that gives us -6 .

Response to task B3 and what students said in support of their answers

For B3 the substitution looks like this: -3(-4)-2(-4). One learner did his substitution like -3-4-2-4 which gave him -13 as his answer. When asked what he did with the multiplication operation of $b p$, he said, he substituted all the numbers and added them together. One learner got -9 as the answer by substituting this way, $3+-4+2+-4=-9$. The other learner substituted correctly, even put the brackets $(-3)(-4)-(2)(-4)=7--2=-5$. When asked what do brackets mean, he said they mean multiply, but he added instead of multiplying. The same learner who got -23 for B2 got -34 - 24 for B3.

\subsection{Section C: Errors associated with simplification of algebraic expressions.}

Table 4: Questions and the participants' responses on the simplification of algebraic expressions

\begin{tabular}{|c|c|c|}
\hline Question Number & Question and Answer & Some Answers Given by Learners \\
\hline C1 & $4 x y+3 x y-3 x-3 x+4-5=7 x y-6 x-1$ & $0 x, 0 x y, 5 x y,(7 x y-6 x+-1), 0-15 x y, x y+x-1$ \\
\hline C2 & $3 a+2 a-6 a+4=--a+4$ & $-5, a=11,15 a, 3 a,-5$ \\
\hline C3 & $2 a b-3 a b+4 a b-6 a b-7 a=-3 a b-7 a$ & $7 a b,-10 a b,-10$ \\
\hline
\end{tabular}

In this section also, the learners did not do well as 3 out of 10 learners who answered this section got the correct answers. For $\mathrm{C} 1$ three learners got final answers of $0 \mathrm{x}, 0 \mathrm{xy}$, and 0 respectively. They simplified until $7 \mathrm{xy}-6 \mathrm{x}-1$ (which is the correct answer), but they 'simplified it further' to get 'one' answer, so $7-6-1=0,0 x y$, and $0 x$. The other learner got $-15 x y$. She argued that from $7 x y-6-1$, she took a negative sign ,because two terms in that three term expression have a negative sign, then she added 7, 6 and 1 , and got $-15 x y$ as the answer.

For $\mathrm{C} 2$ one learner solved for $\mathrm{a}=1$. Another learner got -5 as the answer, $5 \mathrm{a}-10 \mathrm{a}=-5$. It was argued that $\mathrm{a}$ and a cancel each other then -10 and +5 give -5 . The learner who got $15 a$ argued that $5 a+10 a=15 a$. Two learners got $3 a$ as their answer. They got $-a+4$. For them the final answer must not have plus or minus something, it must just be one 
number or one term. So, they argued that when you take -1a from 4 you get $3 a$ and that is the answer.

For $\mathrm{C} 3$, the correct answer is $-3 a b-7 a$. Some learners got $-10 a b$, some got -10 . Again they saw that it does not look right for the final answer to be plus or minus something, so they added -3 and -7 , they got -10ab, the other one got 10 because $a$ and $a$ cancel each other. When asked what happened to $b$, the answer was changed from -10 to $-10 b$.

\subsection{Discussion}

The conceptual framework of our study looks at relational and instrumental understanding of mathematics. We now discuss these results in the light of our theoretical framework. For section A, the learners have the existing knowledge of addition and subtraction of integers. They also know that when given two or more values with different signs, you take the sign of a bigger number. In other words, they have instrumental understanding of basic operations in integers. Skemp (1976) argues that instrumental understanding involves use of rules without reasons. In this situation the learners know that they take the sign of bigger number but do not know why do they that.

Even though the learners have some ideas on basic operations of integers, in section A they made errors when given a task on basic operations of integers, for example, $-65+45=-110$, they added instead and assigned a subtraction sign. This shows they constructed their own knowledge in their minds. This would mean that learners have instrumental understanding of the concept. Skemp instrumental understanding is easier to understand and its rewards are more immediate and more apparent because less knowledge is involved (SAIDE, 2007). We see here that this often leads to erratic thinking. The learners need to have relational understanding to avoid such mishaps.

In section B learners show that they have some understanding of substitution, but lack relational understanding of connecting all the ideas, that bp means $b$ times $p$, so if $b=-3$ and $p=-4$, then bp equals $-3 x-4$ not just $-3-4$. This shows they have instrumental understanding of substitution, they also understand the idea of a place value but they do not understand what to do further.

For section $\mathrm{C}$ the learners believed that the final answer cannot have two or more terms, they said that it does not look good when it has plus or minus something, they wanted the answer to be one value or one term. Learners here also constructed a new (wrong) knowledge in their heads about simplifying. They also lacked relational understanding about simplifying of expressions. Booth (1988) argues that learners face cognitive difficulties in accepting lack of closure. They conceive open expressions as incomplete and tend to finish them. They also see that they need a single termed answer, this comes from arithmetic where addition sign is seen as an action sign which says combine two or more things together and one has a single answer.

There are different types of errors that learners can make when dealing with mathematics. These include systematic errors, constant errors, reading errors, comprehension errors, transformation errors, process skills errors, encoding errors and careless errors (Cox, 1975, Ryan, 2007). Learners do not have to make all these errors at once, or when performing one task. For question A1 and A2, the learners made constant / systematic errors. Systematic errors are defined by Cox (1975) as when there is a repeatedly occurring incorrect response that is evident in a specific algorithmic computation. The learners in question A1 $(27-29)$ got 2 and 3. In question A2 $(-65+45)$ learners got the answers of 110, $-110,120$ and 20. For learners who got 3 and -120 as their answers for questions A1 and A2 respectively, I would say that the type of error they made is a careless error or unsystematic error. Careless errors are defined as those that occur even though the student knew (from a cognitive perspective) exactly how to get a correct answer to the question at the time the incorrect answer was given, and would be expected to give the correct answer when responding to the same question at some later time (Ryan, 2007). The learners have subtracted 27 from 29 and wrote 3 as the answer; and added 65 and 45 and got 120 as the answer. They later gave correct answers and recognised that they made a mistake when they were writing their answers.

Transformation errors were made by the learners when they answered questions A4 to A8. Transformation errors are when the children understand what the question asks but they are unable to identify the operation, or sequence of operations, needed to solve the problem (Ryan, 2007). For (-2) x (-4), the learners gave $-6,6,-8$ as the answers. They took $-2-4=-6$, the other one multiplied negative and negative and got positive, then he added 2 and 4 , the others took the negative sign, then multiplied 2 and 4 to get 8 . This shows that the learners understand what the question asks of them but they do not know what to do to get the correct answer.

Process skills errors were also performed by the learners. This is where learners identify an appropriate operation, but they do not know the procedures necessary to carry out these operations accurately (Ryan, 2007). In question A6, the learners identified the appropriate operation to multiply the signs (- times - times -) which correctly gave then a negative sign. After this they did not know the appropriate procedure to reach the correct final answer. They added the numbers instead of subtracting. The process skills errors are also evident in section B. This is where learners were required to 
substitute in the places of $a, b$ and $p$. Some learners substituted correctly, after substituting they did not multiply where they were supposed to, but they added or subtracted instead, for example, $-3(-4)-2(-4)=3$, what they did was $-3-4-2-4$ $=-13$.

In section C, I see encoding errors performed by the learners. This is where learners see the final answer as incomplete if it has plus or minus something. The learners here give one value or a single termed answer instead of a two term or three term expression. For example for $-a+4$, was simplified further or reduced to 3a. Ryan (2007) defines encoding errors as where the children work out the solution to a problem but could not express this solution in an acceptable written form.

Misconception of overgeneralising of operations is evident in the learners' errors in this study. For $-65+45$ they gave their answer as 110. The other misconception that the learners have in this study is the misconception of interference. This is where the previously learned skills interfere with new skills (Olivier, 1989). "Addition schema is constructed first and is well developed, thus when a question is asked about multiplication, which is more recent (and maybe less secure) piece of learning, the pupil replaces it with a question dealing with earlier (and presumably more securely learned) material (Olivier. 1989, p.11). In questions A4 to A6, the learners were required to multiply. (-2) x (-4). The learners wrote this again as $-2-4$ and gave the answer as -6 . They just added instead of multiplying. $24 x-4$, some learners gave 20 as the answer, they just subtracted 4 from 24 instead of multiplying 24 by -4 . For $-2 x-2 x-4$, learners gave 8 and -8 as their answers, this clearly shows that they added or subtracted the numbers instead of multiplying them. This also happened for question B3 (bp - ap). The learners were required to multiply the values of $b$ and $p$, and those of $a$ and $p$. The learners did not do that, they added the values of $b$ and $p$ and those of $a$ and $p$. The misconception of meaning is also present in the learners in this study. They conceive open expressions as incomplete and tend to finish them (Cobb, 1994). They want to see a one value or a one term answer, an error of conjoining Macgregor and Stacey (1997).

For question A4 (-2) $\times(-4)$, in the beginning we wrote this question as $(-2)(-4)$, but we decided to make the question clear and put the multiplication sign between the brackets because we thought maybe other learners will not recognise that they have to multiply. we was surprised that some learners just added instead of multiplying, because they argue they did not see the multiplication sign. We am now wondering what answers would they have given if we left the question as it was before, (-2) (-4). According to Olivier (1989, p.11) "Addition schema is constructed first and is well developed, thus when a question is asked about multiplication, which is more recent piece of learning, the pupil replaces it with a question dealing with earlier material".

\section{Conclusions}

\subsection{Findings}

We have found different types of errors when these participants answered questions on operations with integers. These are:

- Systematic errors: Learners made systematic errors when answered questions for example for $27-29$, some learners gave 2 as the answer. To the question and for $-65+45$, some learners gave 110 and -110 as their answers. Learners would persist with this line of reasoning.

- Careless errors: This error was also evident in one learner. She gave 3 as her answer to the question. She argued that $29-27$ gave her 3 , but later corrected the mistake and recognised that was an error.

- Transformation errors: These were seen in the learners' answers of multiplication questions. For $(-2) \times(-4)$, some learners gave -6 or 6 . This shows they added or subtracted. For $(24 x-4)$, three learners gave 20 as the answer, showing they worked out $24-4$ instead. For $-2 x-2 x-4$, answers of 8 , and -8 were obtained. For -25 $\div-5$, three learners got -30 and 30 as their answers. For $14 \div-2$, one learner gave 12 as the answer to this question. Thus learners were seen to disregard the multiplication and division operations and stuck to addition and subtraction; operations they have first met and were more familiar with.

- Process skills errors: For $14 \div-2$, one learner gave 7 as the answer instead of -7 . The learner here identified the appropriate operation, but did was unsure of the correct sign (Ryan, 2007). In section B process skills were also seen. This is where learners were required to substitute $a, b$ and $p$ with the given values. For $p+a$ there was correct substitution $-4+2$, but the learner got -6 as the answer. This is related to systematic errors discussed above.

- Encoding errors: These errors were evident in Section C where learners see the final answer as incomplete if 
it has a plus or minus something or if there are more than one term. For example the question -3ab - 7a was 'completed' as 7ab, -10ab or -10 as the final answer.

The above error types are not comprehensive but in our view these were the most eminent. We are also aware that other researchers could term them differently.

We also noted some reasons for the errors. These were:

- Misconception of overgeneralisation: This was seen in the learners' responses to questions $27-29$, two learners gave 2 as the answer. For $-65+45$, learners got 110 or -110 . Olivier (1989) claims that the learner overgeneralise operations. He further states that available research suggests that one guiding principle is children's erroneous conception is that subtraction is commutative.

- Misconception of interference: This was evident in the learners' answers to questions where the learners were required to multiply; $(-2 x-4)$ or to divide $(-25 \div-5)$. Some learners in these situations did not multiply or divide, they added and subtracted instead. This can be due to the previously learned skill (addition and subtraction) interfered with the new skill (multiplication and division). Olivier (1989) argues that misconception of interference occurs when the previously learned skill interferes with the new skill.

- Misconception of meaning: This was evident in the learners' responses of section $\mathrm{C}$. the learners could not make meaning of having a two or a three termed final answer. They wanted to 'complete' it by having a onevalue or a single termed answer. For example, $-\mathrm{a}+4$ was completed as $-3 a$ or -5 .

We find these findings important in that learners cannot be expected to successfully continue with high school mathematics if they are not resolved.

\subsection{Generalizability and limitations of the results}

We did not intend to put a section $C$ on collecting like terms in the study because the study is about the errors made when dealing with operations on integers. But, we did put this section because we wanted to see whether the learners make the same or similar errors that they make when they questions like (i) $22-29+3$ for section A and (ii) $22 x-29 x y+$ $3 x$ for section C. For (i) the answer is -4 , for (ii) The answer given was $-4 x y$. The learners made an error of wanting to have a single termed answer. The learners also made an error of treating $22 x-29 x y+3 x$ as $22-29+3$ ignoring the different variables.

This topic of integers is a difficult topic throughout South Africa and beyond. We think that the results are generalizable and are common among to most grade 9 learners worldwide even though we used a very small sample. In the light of this study, we propose the following recommendations;

\subsection{Recommendations}

- Given that most errors on operations on integers are due to instrumental understanding of the topic, teachers need to use varied resources such as number lines when teaching operations on integers. Teachers can also use stories for learners to visualise operations on directed numbers. These can be used for learners to question whether the answers they give to operations on integers make sense.

- Teachers are urged to use any opportunity to interview and probe learners on their errors. This encourages learners to reflect on their errors and thereby profit from learning from them.

\section{References}

Adams, G. R. \& Schvaneveldt, J. (2011). Understanding research methods. Longman Publishing Group: London.

Bauer, M. (1996) 'The Narrative Interview', London School of Economics and Political Science, Methodology Institute, Methodology Institute:London.

Cobb, P. (1994). Learning Mathematics: Constructivist and Interactionist Theories of Mathematical development. Springer: New York.

Cohen, L., Manion, L. \& Morrison, K. (2002). Research Methods in Education: T. J. International Ltd, Padstow, Cornwall, Great Britain.

Cox, L.S., (1975). Systematic errors in the four vertical algorithms in normal and handicapped populations. In Journal for Research in Mathematics Education. Volume 6. Number 4. National Council of Teachers in Mathematics.

Findon, D. (1998). Comments on "Relational understanding and instrumental understanding" by R Skemp. Mathematics Teaching, 77. Retrieved September 21, 2004, from http://s13a.math.aca.mmu.ac.uk/Student_Writings/Journal/Dominic_Findon2.html

Luneta, K. \& Makonye, P.J., (2010). Learner errors and misconceptions in elementary analysis. A case of a grade 12 class in South Africa. Acta Didactica Napocensia. Volume 3, Number 3.

MacGregor, M. \& Stacey, K. (1997). Cognitive models underlying students formulation of simple linear equations. Journal for Research 
in Mathematics Education, 24(3), 217-232

Nesher, P. (1987). Towards an Instructional Theory. The role of students' misconception. For the Learning of Mathematics: Volume 7. Number 3; FLM publishing Association.

Olivier, (1989). Handling pupils' misconceptions: University of Stellenbosch.

Riccomini, P.J., (2005). Identification and remediation of systematic error patterns in subtraction: Volume 28. Clemson University.

Rolfe, G. (2006). Validity, trustworthiness and vigour: quality and the idea of qualitativ research. Methodological Issues in Nursing Education, 306-310.

South African Institute of Developmental Education (SAIDE): (2007). Open Education Resources Project. DoE: Pretoria

Skemp, R. R. (1976). Relational Understanding and Instrumental Understanding: Department of Education, University of Warwick.

Ryan, W. J. (2007). Children'S Mathematics 4-15: Learning From Errors And Misconceptions: Learning from Errors and Misconceptions. McGraw-Hill Internationa: New York. 